\title{
Protée
}

\section{Fond et forme dans l'image allégorique}

\section{Tony Jappy}

Volume 33, numéro 1, printemps 2005

\section{L’allégorie visuelle}

URI : https://id.erudit.org/iderudit/012263ar

DOI : https://doi.org/10.7202/012263ar

Aller au sommaire du numéro

\section{Éditeur(s)}

Département des arts et lettres - Université du Québec à Chicoutimi

\section{ISSN}

0300-3523 (imprimé)

1708-2307 (numérique)

Découvrir la revue

\section{Citer cet article}

Jappy, T. (2005). Fond et forme dans l'image allégorique. Protée, 33(1), 9-23. https://doi.org/10.7202/012263ar

\section{Résumé de l'article}

Il y a vingt ans, l'historien de l'art américain Craig Owens publiait en deux parties une étude visant à réhabiliter l'allégorie dans un contexte postmoderniste. Cette étude est devenue depuis un passage obligé de toute réflexion sur ce mode. S'inspirant très largement d'un texte de Walter Benjamin consacré au drame baroque allemand, Owens soutenait entre autres que l'allégorie est résolument tournée vers le passé avec, pour figures caractéristiques, la ruine isolée et la mélancolie et que sa structure spécifique peut se concevoir comme le « supplément » derridien.

S'appuyant sur deux séries de photographies allégoriques contemporaines, le présent article cherche à replacer certaines des thèses d'Owens dans un cadre sémiotique peircien en s'intéressant tout particulièrement à sa tentative de caractériser le mode par son contenu et à la thèse du passéisme mélancolique qui lui serait spécifique.
Ce document est protégé par la loi sur le droit d'auteur. L'utilisation des services d'Érudit (y compris la reproduction) est assujettie à sa politique d'utilisation que vous pouvez consulter en ligne.

https://apropos.erudit.org/fr/usagers/politique-dutilisation/ 


\section{FO ND ET FO RME DAN S L'IM AGE ALLÉG O RIQ U E}

TONY JAPPY

The occidental mind has thought in threes since the time of Plato, except in the middle of the twentieth century, when Cartesian France began to cast her spell on other nations. $\quad$ (M. Edwards, 2003: 238)

\section{INTRODUCTION}

De la fragilité des thèses, de la mutabilité des expressions d'art et de l'incompatibilité des théories du signe...

Prenons, par exemple, le cas d'un texte paru il y a un peu plus de vingt ans, en pleine effervescence de la sensibilité postmoderne. L'historien de l'art américain, Craig Owens, publiait alors une étude en deux parties célébrant le retour en force de l'allégorie dans les "visual arts», et ce, aussi bien dans le domaine de la création artistique que dans celui de la critique. Par sa profondeur et par la finesse de ses analyses, l'étude d'Owens est devenue, depuis, un passage obligé de toute réflexion sur ce mode ${ }^{1}$. S'inspirant très largement d'un texte de Walter Benjamin (1985), Owens soutenait entre autres que l'allégorie est autant une attitude, une «impulsion», qu'une technique, une perception autant qu'un processus (1984: 204); que, caractérisée par un certain nombre de figures spécifiques, toute allégorie est résolument tournée vers le passé; que l'image allégorique en particulier se reconnaît à ce qu'elle est moins une invention que la confiscation ou l'appropriation par l'allégoriste de l'image d'un autre; enfin, sur un plan plus formel et dans un langage pénétré d'un certain discours verbocentriste français introduit aux États-Unis sans doute au milieu des années 1960 (voir par exemple Cusset, 2003: 38-42), il affirmait que l'allégorie devait se concevoir comme «supplément», l'assimilant au statut décerné, dans le cadre de la grammatologie, à l'écriture dans ses rapports avec la langue parlée.

La visée de son article est polémique: Owens, comme d'autres historiens et praticiens de l'art contemporain (on pense, par exemple, au photographe Victor Burgin), non seulement consacre cet engouement pour l'allégorie dans l'art de son époque, mais en même temps il règle ses comptes avec le modernisme et ses théoriciens. Sur ce point précis, le sémioticien n'a absolument rien à dire. De plus, 
il serait présomptueux de vouloir revenir sur les jugements, le plus souvent très éclairants et perspicaces aux yeux du non-spécialiste, que portait alors Owens sur les artistes de l'époque (Robert Smithson, Robert Rauschenberg, Cindy Sherman, Sherry Levine, Laurie Anderson et d'autres encore), ainsi que sur les stratégies artistiques spécifiques qu'il décelait dans leur œuvre, jugements qui se cristallisent dans cette remarque qui a si souvent été citée en exemple:

L'appropriation, la spécificité situationnelle, le côté éphémère, l'accumulation, la discursivité, l'hybridation, ces stratégies différentes/diverses caractérisent une grande partie de l'art contemporain et le différencient de ses prédécesseurs modernistes.

(Owens, 1984: 209)

Il s'agit donc, selon Owens, d'un art qui se reconnaît à sa tendance à détourner en quelque sorte les images d'autres artistes, faisant ainsi un pied de nez aux canons traditionnels de l'œuvre unique, de l'authenticité et de l'artiste créateur; à son «sitespecificity", c'est-à-dire son assise situationnelle grâce à laquelle l'œuvre d'art, à l'instar du «land art» de Robert Smithson, est lié organiquement, déictiquement à l'endroit où elle a été créée; à sa manière de cultiver l'impermanence, le fugace, à tel point que, dans certains cas, il ne reste de trace de l'œuvre originale que sous la forme de photographies; à sa tendance à accumuler des objets empruntés à des contextes variés ou détournés d'eux, le plus souvent sans but précis; enfin, à son caractère résolument et consciemment allégorique.

Néanmoins, au vu de certaines thèses empruntées notamment à Walter Benjamin et à divers représentants du mouvement poststructuraliste ${ }^{2}$, on peut légitimement se demander si les caractères spécifiques qu'Owens attribuait à l'allégorie proprement dite ont encore aujourd'hui, c'est-à-dire dans un monde que l'on baptise volontiers "postvisuel», cette même valeur théorique. C'est pourquoi, en examinant à tour de rôle quelques-unes des thèses d'Owens et le cadre théorique à l'intérieur duquel il a conçu son argument, et en s'appuyant sur l'étude d'allégories picturales prises dans l'œuvre de deux artistes contemporains, la présente étude vise à montrer combien il est difficile de codifier, une fois pour toutes, un mode de représentation apparemment aussi protéiforme que l'allégorie visuelle (ou autre) au moyen des divers contenus et inspirations que l'on croit pouvoir lui attribuer. On verra, par la même occasion, que l'invariant de ce mode si particulier, s'il en existe un, ne peut être simplement fonctionnel ni même «stratégique». Dans l'étude que j'entreprends ici, trois questions principales se poseront donc en filigrane:

a) Cette impulsion allégorique se caractérise-t-elle forcément par le passéisme spécifique que lui attribuait Owens? Jusqu'à quel point les caractéristiques de l'allégorie, telles qu'Owens et, avant lui, Walter Benjamin les concevaient, sontelles encore valables aujourd'hui? Certes, il peut paraître bien téméraire de remettre en cause une autorité aussi réputée que Benjamin, mais il y a lieu de penser que le cadre théorique qui ressort de sa belle étude du drame baroque allemand n'a peut-être rien d'universel.

b) D'où vient la conception quasiment linguistique de l'allégorie (l'allégorie visuelle serait un pictogramme, un hiéroglyphe, un rébus)? Sommesnous obligés d'y adhérer? Peut-on poser sous une autre forme cette attribution à l'allégorie du statut de supplément? Autrement dit, quelles différences formelle et fonctionnelle peut-on déceler entre la métaphore comme forme sémiotique et l'allégorie comme mode pictural, textuel (et filmique)?

c) Quel rapport y a-t-il entre texte et image dans le mode allégorique? Quel peut en être le caractère invariant qui lui a permis de venir jusqu'à nous depuis ses origines anciennes? Jusqu'à quel point une réflexion strictement sémiotique sur l'allégorie visuelle peut-elle révéler les limites de l'analyse de l'historien de l'art (sans pour autant invalider ses propos d'ordre artistique)?

Dans ce qui suit, je compte revenir dans un premier temps sur les propos de Walter Benjamin 
qu'Owens a jugés utiles de citer, afin d'en évaluer la pertinence actuelle; je reviendrai également sur un aspect du rapport entre image et texte qui illustre parfaitement, à mon sens, la nature du poststructuralisme dont Owens s'est inspiré, ce qui devrait permettre de mieux saisir la nature de la «dérive» linguistique que prend son analyse. Enfin, après avoir examiné des exemples d'allégories photographiques contemporaines - c'est-à-dire, deux séries allégoriques qui ont été conçues bien après la publication du texte d'Owens - pour montrer que les artistes photographes en question ont été inspirés par d'autres critères que ceux qu'a avancés Benjamin, je reprendrai, dans le cadre de la sémiotique peircienne, la problématique du prétendu universel invariant dans l'allégorie en opposant celle-ci à la métaphore.

\section{LA DETTE ENVERS WALTER BENJAMIN}

Comme le note Owens, le renouveau de l'allégorie comme mode artistique et littéraire coïncide avec le regain d'intérêt porté à la pensée de Walter Benjamin, dont il dit que c'est le seul critique du XXe siècle à traiter du mode sans préjugé, de manière philosophique (Owens, 1984: 204). L'ouvrage que cite Owens, Origine du drame baroque allemand (1985), conçu par Benjamin dans l'intention de demander son habilitation à enseigner à l'université, est composé de quatre chapitres, dont le troisième s'intitule justement "Allégorie et trauerspiel» ${ }^{3}$ et lui fournit un certain nombre de traits qui caractérisent à ses yeux l'allégorie contemporaine. Bien que certains de ces traits paraissent encore aujourd'hui très pertinents, d'autres soulèvent des problèmes, notamment le statut universel qu'il croit bon de prédiquer, d'une part, du principe du regard mélancolique de l'artiste allégoriste; d'autre part, des deux emblèmes clés de l'allégorie, à savoir les figures de la ruine et de la tête de mort. Voyons en deux points de quoi il ressort.

Le premier concerne les caractères prétendument spécifiques de la création allégorique dans les arts visuels. Dans une discussion de l'œuvre d'un contemporain, l'artiste Troy Brauntuch, et en faisant preuve de convictions essentialistes assez surprenantes,
Owens affirme que le tempérament de l'allégoriste se reconnaît à sa tendance à porter sur les choses du monde un regard mélancolique: "Celui de Brauntuch est donc ce regard mélancolique que Benjamin a assimilé au tempérament allégorique»(Owens, 1984: 205-206). Il cite à cet égard un extrait du texte de Benjamin qui va par ailleurs au cœur du problème de la composition artistique de l'allégorie:

Si l'objet devient allégorique sous le regard de la mélancolie, celleci lui enlève la vie, il demeure comme un objet mort, mais assuré dans l'éternité, et c'est ainsi qu'il se présente à l'allégoriste, livré à son bon plaisir. Voici ce que cela signifie: il sera désormais hors d'état d'émettre une signification, un sens; il n'a d'autre signification que celle que hi donne l'allégoriste. Celui-ci la dépose en hui, dans un geste de condescendance: voilà sa réalité, non pas psychologique mais ontologique. Dans la main de l'allégoriste, la chose devient autre chose, il parle ainsi d'autre chose, elle devient pour lui la clé du domaine du savoir caché, l'emblème de ce savoir auquel il rend hommage. Voilà ce qui fait de l'allégorie une écriture. (Benjamin, 1985: 97)

Selon Owens et Benjamin avant lui, l'un des caractères spécifiques, quasiment définitoires de l'allégorie, serait donc ce regard mélancolique qui offre à l'allégoriste le pouvoir de prendre des objets dans des contextes divers et, en les re-contextualisant à l'intérieur de son image, de les dépouiller de leur identité «sémantique» propre, originaire, et de leur attribuer, dans son œuvre, une signification tout à fait autre. Si l'on n'éprouve aucune difficulté à reconnaitre le souffle allégorique dans cette aptitude à recomposer dans un cadre nouveau des objets ainsi décontextualisés, il en va autrement de l'universalité du regard mélancolique. C'est une notion à première vue séduisante, mais gare aux contre-exemples quand on avance des principes universels. Or, comme on le verra dans le cas du principe en question, les contreexemples ne manquent pas, et Owens semble avoir pris pour attribut universel ce qui relève plus spécifiquement du baroque allemand.

Il faut accueillir avec la même prudence deux autres prétendues caractéristiques de l'allégorie dans les arts visuels, à savoir, d'une part, l'attraction pour le 
fragmentaire, l'imparfait et l'incomplet qu'est censé ressentir l'allégoriste, attirance qui s'exprime pour Owens, comme pour Benjamin, dans le culte de la ruine ${ }^{4}$ :

L'allégorie est invariablement attirée par le fragmentaire, l'inachevé, l'incomplet, affinité qui trouve son expression la plus complète dans la figure de la ruine que Benjamin identifie comme l'emblème allégorique par excellence. (Owens, 1984: 206)

D'autre part, la pratique artistique qui consiste à accumuler dans la composition objets et fragments d'objets de façon aléatoire, sans but précis, pratique qu'affectionnent, selon Owens, les spécialistes du photomontage:

Nous devrions [...] aussi nous attendre à rencontrer une motivation allégorique dans le photomontage, "car le point commun de [l'allégorie], c'est qu'elle accumule sans cesse des fragments, sans but rigoureusement défini». (Ibid.: 207)

En attendant d'examiner plus en détail justement quelques exemples de photomontage, on peut déjà avancer qu'Owens aussi bien que Benjamin ont procédé par induction abusive. Car ce qui se profile derrière les caractéristiques de l'allégorie en question, c'est tout simplement la remarquable gravure de Dürer de 1514, Melencolia 1 (planche 1), œuvre à laquelle Benjamin consacre les dix dernières pages de son deuxième chapitre, en prélude en quelque sorte à l'étude étendue qu'il entreprend dans son chapitre trois du thème de l'allégorie et du trauerspiel.

Certes, on y voit un ange maussade au regard farouche qui symbolise le mélancolique, le génie, ou encore l'artiste qui découvre avec effroi les limites de son inspiration. Mais, comme nous le rappelle l'un des nombreux commentateurs de cette œuvre, l'esprit allemand qui a produit Dürer et la Réforme nous a également donné la psychanalyse (Clark, 1969: 155). Autrement dit, l'interprétation de la gravure ne peut faire une impasse sur la vision forcément personnelle d'un artiste de génie qui travaillait à une période historique et dans une localisation géographique bien précises. De plus, si l'on est arrivé avec le temps à attribuer à la gravure une interprétation plus ou

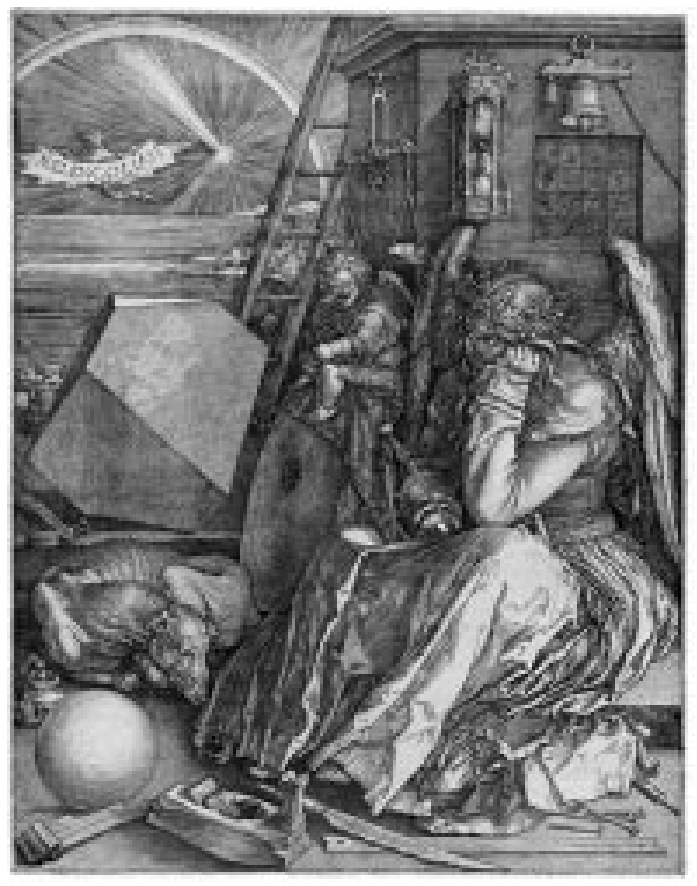

Planche 1. Albrecht Dürer, Melencolia 1, 1514.

moins stable, c'est précisément parce que les attributs du mélancolique, qui pour Benjamin semblent être disposés çà et là sans but précis, contribuent en réalité à une composition dont les divers éléments ne s'interprètent pas tous sur le même plan; et à conclure que l'œuvre est la parfaite illustration d'une certaine conception du génie artistique, c'est consigner, dans une proposition relativement simple - «Le génie est un ange maussade», par exemple, ou, à la Benjamin, «Le créateur est un mélancolique» -, tout un travail d'inférence, sorte de somme fibrée où tous les éléments de la gravure coopèrent pour faciliter collectivement ce jugement. Sur ce premier point, par conséquent, il apparaît que l'historien de l'art n'est guère convaincant.

Le deuxième point, qui a trait aux emblèmes types de l'allégorie, n'est pas moins problématique que le premier. Ruine et art plastique sont, selon Owens, intimement associés: «Ici les œuvres de l'homme sont réintégrées au paysage; c'est ainsi que les ruines figurent l'histoire comme processus irréversible de dissolution et de décadence, une distanciation progressive de l'origine» (Owens, 1984: 206). Ici aussi, Owens se réfère à Benjamin:

[...] dans l'allégorie, c'est la facies hippocrata de l'histoire qui s'offre au regard du spectateur comme un paysage primitif pétrifié. L'histoire, dans ce qu'elle a toujours eu d'intempestif, de douloureux, d'imparfait, s'inscrit au visage - non: dans une tête 


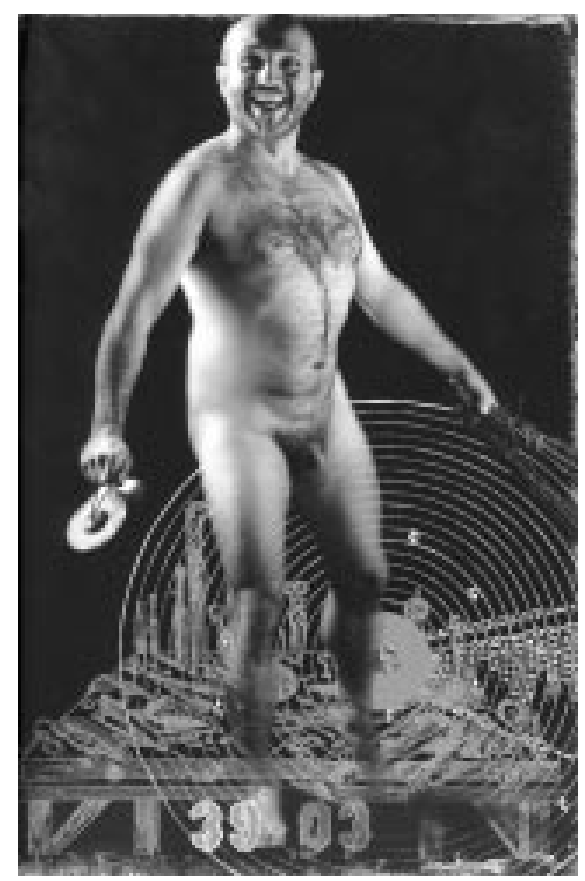

Planche 2. Peter Greenaway, Marsyas (1995).

de mort. Et aussi vrai qu'il n'y a en celle-ci nulle liberté «symbolique» de l'expression, nulle harmonie classique de la forme, nulle humanité, l'énigme qui s'exprime dans cette figure, la plus soumise à l'empire de la nature, ce n'est pas simplement la nature de l'existence humaine, mais l'historicité de la biographie individuelle. C'est là le noyau de la vision allégorique, de l'exposition baroque de l'histoire comme histoire des souffrances du monde; elle n'a de signification que dans les stations de sa décadence. (Benjamin, 1985: 178-179)

C'est donc la facies hippocratica, ce visage creux et blême du mourant gisant sur le lit de mort, qui serait l'expression la plus fidèle de la soumission humaine à l'empire et à l'emprise de la nature: l'être humain naît pour mourir, et la tête de mort, lugubre momento mori, serait l'emblème le plus authentique de notre humanité.

Nul doute que la ruine ait pu, puisse encore présenter la valeur symbolique de la dissolution et la décadence induites par le passage inéluctable du temps - citons le «landart» de Robert Smithson dont Owens parle souvent, ces installations-sculptures qui finissent toujours par être récupérées par l'environnement naturel dans lequel elles ont été exécutées, dans les eaux du lac à Salt Lake City, par exemple -, ni que la tête de mort ait pu servir, surtout dans les églises, de rappel de leur mortalité aux chrétiens peu enclins à suivre le droit chemin. Il

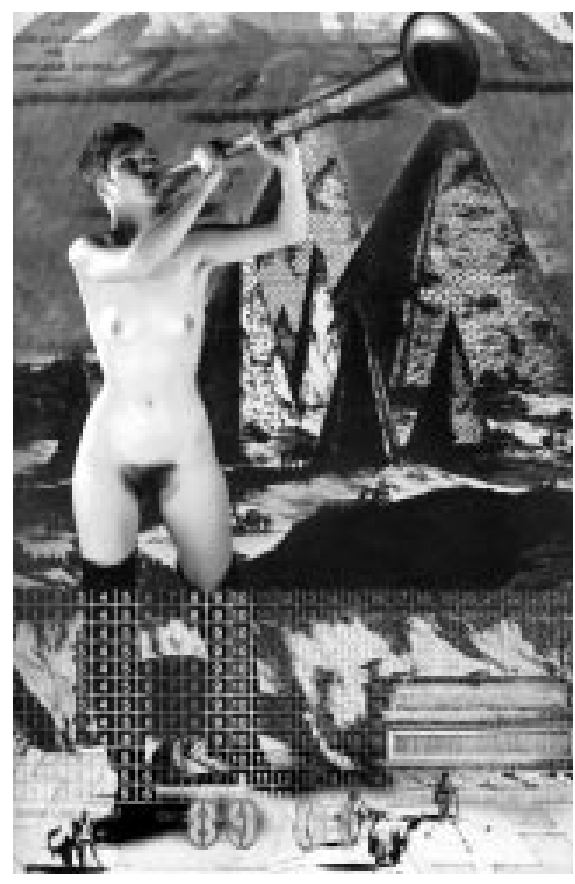

Planche 3. Peter Greenaway, La Destinée (1995).

n'empêche que les allégoristes ont pu célébrer bien d'autres aspects non moins fondamentaux de la condition humaine. Négliger ce fait, comme c'est le cas chez Craig Owens, et promouvoir la tête de mort et la ruine en emblèmes privilégiés de l'allégorie, c'est restreindre considérablement la portée de ce mode si particulier, reléguer bien des allégoristes à un statut subalterne et sacrifier inconsciemment ses instincts critiques à un mouvement de pensée d'origine européenne, francophone et vaguement iconoclaste.

\section{CENT ALLÉGORIES POUR REPRÉSENTER LE MONDE}

Pour comprendre encore mieux la fragilité de cette conception postmoderne de l'allégorie, examinons deux exemples de l'inspiration allégorique relativement contemporaine du cinéaste britannique Peter Greenaway ${ }^{5}$ (planches 2 et 3).

On connait l'histoire de Marsyas. Joueur de flûte trop sûr de son art, il défia le dieu Apollon à un concours musical qu'il perdit. Pour le punir de sa témérité, Apollon l'écorcha vif de ses propres mains. Dans l'imaginaire greenavien, Marsyas est l'allégorie de la musique comme force perturbatrice (Greenaway, 1998 : 244). Pour le représenter, l'artiste fait porter au modèle de petites cymbales et des brindilles de bouleau, métonymies des cuivres et des percussions. En guise de mauvais présage, l'image «importée» de la charte de la musique céleste, de Robert Fludd, est 
centrée sur une représentation de la table de dissection avec son bel assortiment d'instruments, empruntée au manuel - ou plutôt détournée du manuel d'enseignement de l'anatomiste Vésale, paru au XVI ${ }^{\mathrm{e}}$ siècle. Le texte inscrit sur le disque est orienté à trois heures du matin, dont Greenaway affirme que c'est l'heure la plus triste de la nuit. Marsyas souriant et totalement nu est saisi dans une attitude d'hilarité innocente avant son épreuve avec le dieu. Malgré le fait que son sujet va connaitre un sort des plus tristes, il est difficile de croire que l'épisode, ainsi représenté, ait été conçu sous le coup de la mélancolie. Et bien que certaines des figures des allégories de la série portent ce qui pourrait être pris pour une tête de mort - dans le cas de l'allégorie 4, Junon, par exemple, il s'agit en fait d'un «masque théâtral de l'héroïne tragique, l'emblème de son emploi à venir, emprunté au magasin d'accessoires de l'Opéra de Strasbourg» (ibid.: 220) -, les masques en question semblent avoir été incorporés à l'image autant pour leur potentiel plastique que pour leur valeur symbolique.

Qu'en est-il de la ruine, cet autre emblème-clé de l'allégorie? À l'image de la Fama de Virgile, la Destinée de Greenaway en est peut-être un exemple (planche 3). Elle se déplace sur la terre la tête dans le ciel. Le fond de l'image est fourni par une gravure de Kircher représentant des pyramides égyptiennes. Comme le note Greenaway, l'illustrateur n'est de toute évidence jamais allé en Égypte, car les pyramides sont trop pointues et le matériau employé dans leur construction est visiblement celui de l'Europe du Nord, à savoir de la brique. Par ailleurs, l'image a été délibérément inversée afin de donner l'impression qu'elle est vue dans un miroir. En voici l'explication de Greenaway: «Dans ce décor scénique fantastique, la figure du Temps-Destin se pavane en cuissardes, soufflant dans sa trompette et immergée jusqu'aux genoux dans le fleuve des jours qui passent, même s'il ne s'agit que d'un calendrier prévu pour un usage exclusivement bancaire comme l'indique l'inscription" (ibid.: 272).

Même si l'on peut croire déceler ici et là les avatars de certains des caractères de l'allégorie mis en avant par Owens et, avant lui, par Benjamin, il est néanmoins manifeste que l'inspiration de Greenaway et l'intérêt qu'il porte à ces thèmes sont ailleurs, car s'il fallait, à partir de ce corpus si personnel et si particulier, dégager les traits définitoires du mode, nous serions obligés d'inventorier l'ironie du regard de l'allégoriste, la nudité des modèles, l'exubérance chromatique des images (qu'aucune reproduction en noir et blanc n'est capable de rendre) et, malgré tout, le sérieux de l'entreprise. L'ironie surtout, car, en nous définissant comme spectateurs devant cet éventail de corps nus et la réalité détaillée de leur sexualité, Greenaway nous installe dans le rôle de voyeurs (Gasquet, 2003). Dans un érotisme qui paraît volontairement ambigu, hommes, femmes, jeunes et moins jeunes, des enfants aussi, tous habillés par des "décors» somptueux, sont livrés à notre regard fasciné, comme si Greenaway cherchait à se jouer de la moralité conventionnelle: cette image du TempsDestin relève-t-elle de la porno soft? De plus, les commentaires proposés en fin d'ouvrage pour expliquer les attributs associés aux figures développent cette même ambiguité.

D'autre part, il est clair que Greenaway, en artiste émerveillé par le visuel, prend plaisir à explorer et à exploiter les possibilités créatrices apportées au photocollage par la déjà sophistiquée technologie de la numérisation ${ }^{6}$; et l'ouvrage vérifie clairement ce que Benjamin a bien reconnu comme la tendance à décontextualiser des objets (surtout des images) pour les recomposer dans un nouveau contexte avec un sens différent. Mais, malgré le plaisir évident que Greenaway prend à construire ce microcosme - son mundus subterraneus à lui -, l'entreprise est sérieuse: l'artiste avoue s'intéresser à des archétypes et, à partir de l'individuel, les cent allégories qu'il nous propose réussissent à représenter l'universel, une typologie humaine signalée par l'attribut le plus clairement reconnaissable, à savoir le sexe. Il s'agit en quelque sorte d'une nouvelle Iconología, conçue par un Greenaway-Ripa sans gravitas mais néanmoins sérieux, au point où cent cinquante habitants de Strasbourg, visiblement éduqués et intelligents (une 
fois le choc de la nudité frontale passé, ce qui frappe dans les images, c'est la sensibilité et l'intelligence qui se lisent dans les regards), ont accepté de se livrer nus à l'appareil des deux photographes et à leur directeur artistique. Enfin, tout en jouant avec les conventions du mode, comme on le verra plus loin, Greenaway n'en respecte pas moins la forme pour nous offrir une célébration païenne de la beauté du corps humain.

\section{PeINTURe et ÉCRITURe: Vers L'ICONOClasme}

Pour comprendre comment Owens a pu épouser ce qui revient à une théorie linguistique de l'image, qui conviendrait davantage aux poésies concrètes des Lettristes qu'à l'œuvre de Greenaway et de l'autre artiste photographe qu'on étudiera plus loin, je voudrais examiner un aspect de l'évolution de l'art au siècle dernier, plutôt que d'essayer de retracer le développement du poststructuralisme sur le sol américain, car cette évolution illustre bien le climat intellectuel et artistique par lequel Owens a pu être influencé dans sa description de l'allégorie visuelle.

D'abord, les concepts. C'est dans ces termes que se poursuit la description de l'allégorie:

[...] Ce que révèle [la réciprocité que l'allégorie établit entre visuel et verball, c'est la nature essentiellement pictographique de l'œuvre allégorique. Dans l'allégorie, l'image est un hiéroglyphe, l'allégorie est un rébus, une écriture [writing] composée d'images concrètes. (Owens, 1984: 208-209)

Ce qui frappe dans cette définition de l'allégorie visuelle, c'est l'accumulation de références à l'écriture - gram-, -glyph, writing -, autant de termes qui vident l'image de son contenu iconique là précisément où la tradition préférait le terme d' "emblème» car, comme nous l'avons vu, Owens nous précise bien que c'est l'allégorie dans les arts visuels qu'il privilégie dans son article. Ici aussi il reprend une idée de Benjamin déjà évoquée plus haut:

Dans la main de l'allégoriste, la chose devient autre chose, il parle ainsi d'autre chose, elle devient pour lui la clé du domaine du savoir caché, l'emblème de ce savoir auquel il rend hommage. Voilà ce qui fait de l'allégorie une écriture. (1985: 97)
Mais à ce stade de son essai, Owens évoque d'autres sources, notamment Barthes, Lacan, Derrida, Paul de Man, à savoir des penseurs européens majoritairement français, sans formation linguistique particulière soit dit en passant, dont l'influence s'est développée considérablement pendant les années 1970 sur le continent américain et à laquelle se sont ralliés bon nombre d'artistes et de critiques d'art de l'époque comme Owens. Démontrer le caractère antivisualiste et "pro-linguistique" de ce mouvement dépasserait le cadre de cet article, mais ses origines et son développement en France pendant le siècle dernier ont été minutieusement retracés dans l'étude monumentale de Martin Jay (1994) à laquelle le lecteur intéressé est renvoyé. En revanche, il est éclairant de voir comment ce même mouvement, auquel participaient Barthes et les autres penseurs mentionnés plus haut, a pu influer sur l'évolution des pratiques artistiques paradoxalement iconoclastes au cours du même siècle, notamment sur le continent américain, et comment ce même mouvement a pu être absorbé et recyclé aussi bien par les artistes que par les critiques d'art.

Considérons, pour commencer, ce tableau de Picasso de 1912, œuvre qui a été exécutée juste après que Ferdinand de Saussure ait assuré son dernier cours de linguistique générale - il y avait décidément quelque chose dans l'air du temps - et qui illustre l'une des grandes innovations artistiques du siècle dernier, à savoir le collage, c'est-à-dire le fait de coller sur la surface plane du tableau des artefacts divers morceaux de journal, de papier peint, des bouts de tissus, etc. - pour en troubler et en subvertir le caractère bidimensionnel. C'est le cas de l'image de Picasso, où des fragments de journal se trouvent là sur la toile dans leur fonction propre d'objet quotidien, mais aussi découpés, de sorte que leur forme évoque celle d'un autre objet quotidien, si bien que les morceaux de journal se substituent et s'allient tout à la fois au travail du pinceau traditionnel (planche 4).

Mais la rupture avec la tradition se manifeste non seulement dans l'adjonction à l'image de quelques morceaux du réel, mais aussi dans cette cohabitation 


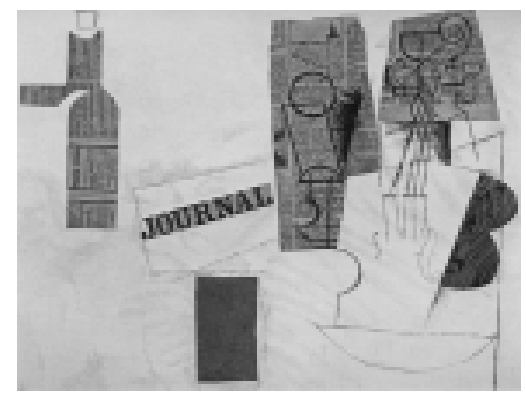

Planche 4. Picasso, Bouteille, verre et violon, 1912. Moderna Museet, Stockholm, Suède. (C) Succession Picasso Paris/sodrac (Montréal) 2005.

alors insolite du linguistique - le légisigne JOURNAL - dans un espace traditionnellement dévolu à l'iconique, pour y créer, grâce au caractère autoréférentiel du mot lui-même et à la tension induite par son statut sémiotique, une nouvelle dimension, une irruption indiciaire déstabilisatrice dans l'œuvre d'art. Au lieu de se tenir en dehors de l'espace pictural sous forme de légende ou à l'intérieur comme signature de l'artiste ou encore comme élément de la représentation illusionniste elle-même le et in arcadia ego de Poussin, par exemple -, l'élément linguistique affirme ici son droit à l'esthétisation. Il s'agit en fait d'une propriété fondamentale de tout signe linguistique dont rend compte le théorème peircien de l'implication iconique, où il est affirmé que tout indice implique un ou des éléments iconiques et, de la même façon, que tout symbole implique un ou des éléments indiciaires, si bien que, par transitivité, tout symbole implique un ou des éléments iconiques (couleurs, forme, texture etc.) (Peirce, 1931-1958: 2.247-249). Dans son tableau de 1912, Picasso tire donc parti des qualités proprement iconiques du mot JOURNAL, de ces configurations spécifiques de qualisignes inhérentes à tout légisigne, qu'il soit indiciaire ou symbolique, pour créer certes une rupture avec la tradition, mais en même temps une œuvre picturale non photographique qui est néanmoins déictique: ce légisigne «dit» le réel au lieu d'en constituer la représentation picturale «muette». Et avec ces deux dimensions iconiques et indiciaires, le tableau constitue, sur le plan proprement sémiotique, un signe complexe où l'iconicité du petit texte est en relation tensive, dialectique, avec celle de l'image sur laquelle il est collé.

Avant la fin du siècle dernier, et ce, précisément à l'époque où Owens composait son article sur l'allégorie, cette tension était en voie de résolution au profit de l'écriture, comme le montre cette image de

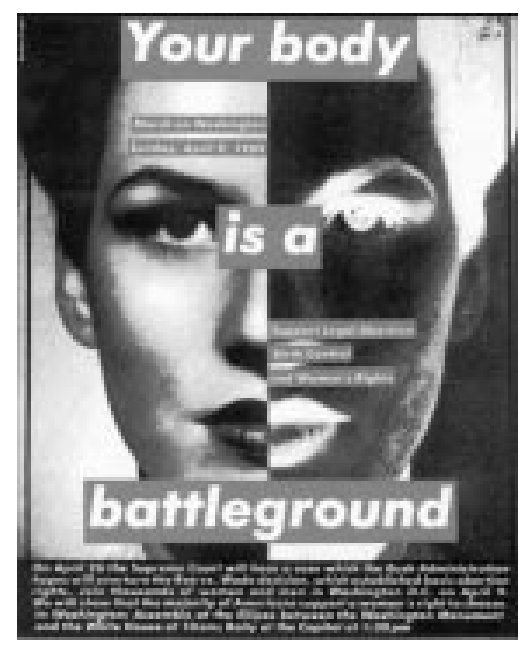

Planche 5. Barbara Krüger, Untitled, 1989.

(c) Barbara Krüger/sOdRAC (Montréal) 2005.

Barbara Krüger, artiste photographe américaine qui s'est forgé une réputation principalement pendant les années 1980. Il s'agit d'une affiche qui date de 1989 et qui invite femmes et hommes à se rassembler et à manifester, afin de protéger le droit des femmes à l'avortement libre, droit que l'administration Bush de l'époque cherchait à leur enlever (planche 5).

L'image se compose de barres de texte blanc sur fond rouge (sorte de cri de guerre écrit en gros, accompagné d'informations pratiques en caractères plus petits), le tout "collé» comme de la peinture de guerre sur un fond formé de l'image d'une tête de femme des années 1950 coupée en deux: à droite le négatif, c'est-à-dire la photo en puissance, à gauche la photographie partiellement développée. On constate facilement que texte et image sont tous deux informés par l'iconicité métaphorique, à laquelle Peirce attribue la forme d'un parallélisme ${ }^{7}$. C'est ainsi que dans le texte s'établit le parallèle entre, d'une part, une bataille et un lieu stratégique à conquérir (battleground) et, d'autre part, la femme et le droit de disposer librement de son corps (your body). Quant à l'image, le négatif et le cliché partiellement développé y signifient que la femme n'assume pas encore totalement son destin, qu'elle n'est que partiellement maîtresse de ses actes puisqu'elle dépend encore du bon vouloir d'une sorte de patriarcat, symbolisé dans cet épisode de la guerre des sexes par George Bush et son administration.

Mais plus significatifs encore sont, d'une part, la hiérarchie qui règle les rapports associant texte et image dans ce photomontage, d'autre part, le procédé 
artistique qui consiste à photographier une photographie, car tous deux révèlent l'ascendance du linguistique dans les représentations picturales, qui s'est développée tout au long du siècle depuis le tableau de Picasso examiné plus haut. En effet, comme dans la plupart des œuvres de cette artiste photographe d'ailleurs, tout se passe dans l'image, comme si le texte avait été disposé ainsi pour «museler» dans l'espace pictural les éléments iconiques, pour en entraver la liberté plastique, pour ainsi dire, pour leur assigner un statut subalterne. Mais cette impulsion iconoclaste va plus loin. Car, en présentant la photographie d'une photographie prise trente ans auparavant - en reléguant à deux degrés de distance le rapport indiciaire au modèle original -, Krüger semble avoir délibérément enlevé à l'image photographique son caractère déictique pour le réinjecter dans le slogan linguistique, ce fragment de langue qui, lui, est collé dessus, car il n'y a guère de photocollages de Krüger dont le slogan ne contienne pas au moins un élément déictique sous la forme d'un pronom personnel ou d'un adjectif possessif. En d'autres termes, l'iconoclasme pratiqué par Barbara Krüger aboutit à la suppression, dans la photographie comme médium, de ce qui constitue son caractère sémiotique définitoire, à savoir son indexicalité.

C'est ainsi que le débrayage déictique opéré systématiquement par Krüger lui permet d'assigner à la composante proprement iconique un nouveau statut plastique afin de mieux la contrôler. Et c'est ainsi que, dans un mouvement qui marque la progression inéluctable de la métaphore de la langue dans notre métaphysique occidentale, l'influence du linguistique semble avoir pris le dessus dans une œuvre dont le mode de représentation photographique est originairement indiciaire et visuel, mais où ne subsiste que son résidu iconique. Et c'est ainsi aussi que cette image, qui est structurée par la métaphore sur deux plans - le linguistique et le visuel-, peut être considérée, dans la perspective adoptée dans cet article, comme l'allégorie non pas de l'évolution du rapport entre texte et image dans l'espace plastique, mais sur un plan plus général: l'allégorie du renversement par le tournant linguistique de la métaphysique visualiste qui fondait notre appréhension du monde depuis l'Antiquité. Remplacer la tyrannie de l'œil par la tyrannie du discours...

Or, c'est dans ce contexte iconoclaste du poststructuralisme triomphant, si «éloquemment» illustré par l'affiche de Barbara Krüger, qu'Owens a conçu sa théorie de l'allégorie, d'où ces références presque innocentes à l'écriture. Restent donc le problème du caractère «supplémental» qu'attribue l'auteur à l'allégorie, et la manière dont s'effectue dans le texte le passage au «tout écrit».

Prenant position contre la distinction opérée par la critique romantique entre symbole et allégorie, il cite, pour le dénoncer, l'extrait d'un texte de Croce: «Cette allégorie qui nous arrive attachée post festum à une œuvre achevée/terminée ne modifie pas l'œuvre d'art. Qu'est-elle, donc? C'est une expression plaquée extérieurement sur une autre expression" (Owens, 1984: 215) ${ }^{8}$. Contre cette conception, et contre le modernisme qui la cautionne, Owens s'insurge:

Au nom de la «justice», donc, et afin de préserver le caractère intuitif de toute ouvre d'art, y compris de l'œuvre allégorique, l'allégorie se conçoit comme un supplément, c'est-à-dire une expression ajoutée extérieurement à une autre. Nous voici devant la stratégie permanente de la théorie occidentale de l'art qui élimine de l'œuvre tout ce qui remet en cause sa détermination comme unité de "forme» et de "contenu». (Ibid.)

Mais loin de se dissocier de cette conception apparemment dévalorisée de l'allégorie, Owens, au contraire, la reprend à son compte, s'en servant pour contester les canons du modernisme, canons dont les artistes de l'époque faisaient allégrement litière, et il avance la notion de supplément justement pour en dégager les «vertus»:

Il est vrai que la signification allégorique paraît supplémentaire. L'allégorie est une extravagance, une dépense de valeurs surajoutées. Elle vient toujours en sus. Néanmoins, le supplément allégorique est non seulement ajout mais aussi substitut. Il remplace une signification antérieure qui s'en trouve ainsi effacée ou cachée. Parce que l'allégorie usurpe son objet, 
elle comporte un danger qui est le risque de le pervertir, de donner à ce qui est seulement ajouté le statut de l'essence. D'où la véhémence avec laquelle l'esthétique moderne, l'esthétique formaliste en particulier, s'en prend au supplément allégorique, car il remet en cause le bien fondé des bases sur lesquelles repose l'esthétique. (Ibid.)

Enfin, emboîtant en cela le pas à Jacques Derrida dans sa critique du penchant logocentrique de Ferdinand de Saussure (Derrida, 1967: chap. 2), Owens effectue, par le biais du concept de supplément, l'assimilation à l'écriture de l'allégorie emblématique, c'est-à-dire visuelle, de la tradition:

Si l'allégorie s'assimile à un supplément, elle s'aligne aussi sur l'écriture dans la mesure où l'écriture se conçoit comme supplément à la parole. Ceci s'inscrit bien sûr dans la même tradition philosophique qui subordonne l'écrit à la parole et l'allégorie au symbole. Il est possible de démontrer dans une autre perspective que la suppression de l'allégorie est identique à celle de l'écrit, car l'allégorie visuelle ou verbale est essentiellement une forme d'écriture - ceci est à l'origine de l'emploi qu'en fait Benjamin dans Origine du drame baroque allemand: «le regard aigu de l'allégorie transforme d'un seul coup les choses et les ouvres en un écrit stimulant». (Owens, 1984: 215-216)

En attendant d'examiner de plus près les conséquences d'une telle conception linéaire et séquentielle de l'allégorie et de la comparer à une conception plus «verticale» des phénomènes en jeu, nous allons examiner deux œuvres d'une artiste française qui a fait de l'allégorie photographique son mode de prédilection.

\section{LES Filles DE RIPA, OU LE MONDE À L'ENVERS}

Cesare Ripa, c'est ce fils de Pérouse qui, à la fin du $\mathrm{XVI}{ }^{\mathrm{e}}$ siècle, fit paraître son Iconología, traité destiné principalement à fournir aux poètes, aux prédicateurs, aux orateurs des sujets allégoriques, métaphoriques et mythologiques divers, mais qui, par la même occasion, est vite devenu un traité d'iconographie pour les artisans de la représentation visuelle du monde (peintres, sculpteurs, décorateurs de théâtre, etc.). Les deux premières éditions de l'ouvrage $(1593,1602)$ ne comportaient que des textes descriptifs. À partir de l'édition de 1603, sans doute devant la franche réussite de l'ouvrage, ces textes s'accompagnaient d'illustrations xylographiques apparemment conçues à partir de dessins qu'aurait fournis le Chevalier d'Arpin, peintre très à la mode à l'époque.

Depuis 1998, l'artiste photographe parisienne Dany Leriche reprend le concept de Ripa en le retravaillant dans un cadre bien à elle, les quelques allégories déjà construites se trouvant consignées pour leur essentiel dans le catalogue de l'exposition de 2000, Les Filles de Ripa ${ }^{9}$. Marie-Domitille Porcheron, qui a préfacé ce catalogue, résume bien le mode de travail très particulier de l'artiste:

[Dany Leriche] choisit donc dans le traité de Ripa des vertus, des vices, des passions, des affects, des concepts, des entités immatérielles qu'illustrent des figures de femmes uniquement, qu'elle confie à des historiens, des historiens de l'art, des écrivains, des philosophes, des artistes... Elle leur demande de choisir un de ces thèmes [...] sur la liste qu'elle a préétablie, à charge pour eux d'inventer ou de compiler les formes textuelles des allégories contemporaines [...]. Bref, Dany Leriche leur demande de fabriquer une Iconologie d'aujourd'hui. (2000: 4)

Il s'agit donc, dans une certaine mesure, d'une collaboration où, dans la plupart des cas, les textes sont mis au point et fournis à l'artiste avant le travail de prise de vue (dans le catalogue de 2000, il y a deux fois plus de textes que d'images). Le projet est par conséquent inachevé, les allégories s'ajoutant à la collection selon la disponibilité des modèles et du nombreux personnel technique impliqué dans l'œuvre; mais il devrait, à la longue, se concrétiser définitivement sous la forme d'un dictionnaire iconographique. Et, on le verra, avec l'œuvre de Dany Leriche, on passe d'un militantisme photographique, structuré à l'occasion par la métaphore, à un militantisme qui opte résolument pour le mode allégorique.

Étant donné que ce sont d'autres que l'artiste photographe elle-même qui choisissent les thèmes et, avant même les prises de vue, en proposent l'interprétation, on pourrait être tenté de croire que 

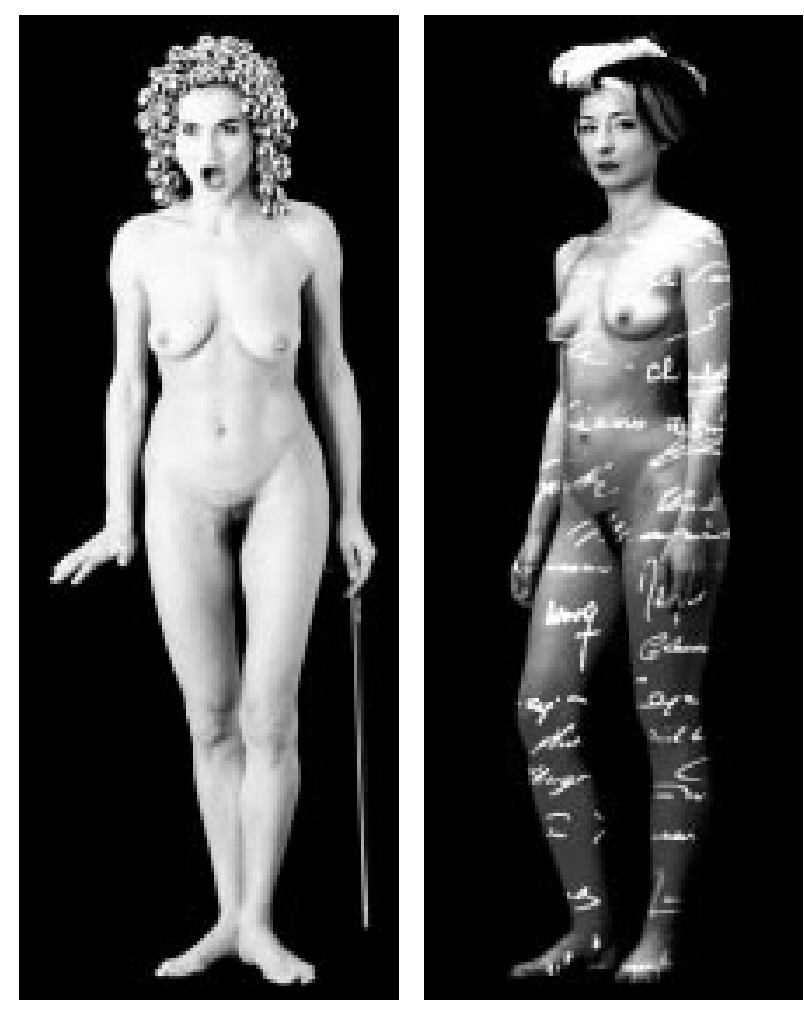

Planche 6. Dany Leriche, Anoki (1998).

Planche 7. Dany Leriche, Sophie (1998).

l'inspiration artistique de ce "collaboratoire» insolite manquerait d'homogénéité et de sens directeur. Rien ne serait moins vrai, car par leurs qualités proprement artistiques et la manière dont les thèmes sont figurés, Les Filles de Ripa offrent, du monde contemporain, une vision de militante féministe à la fois sérieuse, subtile et ironique ${ }^{10}$.

Pour illustrer cette œuvre allégorique si personnelle, examinons deux images extraites de la série des cinq consacrées aux Muses. À la différence de la majorité des allégories, le texte associé a été composé après les prises de vue, ce qui a l'avantage de laisser clairement apparaittre la vision personnelle de l'artiste ainsi que les caractéristiques propres à ses allégories.

Anoki, coiffée d'une toison de grelots dorées et tenant à la main un archet, est photographiée nue, en pose frontale. Le corps est tendu et le visage de la jeune femme se crispe dans un effort vocal: très différente du Marsyas de Peter Greenaway, elle est l'allégorie de la Musique (planche 6).

Dans l'un des rares photomontages à allier prise de vue argentique et traitement numérique, Sophie l'écrivain, coiffée quant à elle d'un chapeau emplumé de velours, porte sur son corps nu la signature de nombreuses personnalités féminines: elle personnifie ainsi la Littérature ${ }^{11}$ (planche 7). Première caractéristique à la fois fascinante et dérangeante de l'œuvre de Leriche: tous les modèles sont, tout comme l'artiste photographe elle-même, de sexe féminin; deuxième caractéristique non moins surprenante, les femmes et les filles ainsi fixées sont toujours nues: des femmes nues mises en scène et photographiées par une femme; troisième caractéristique de toute l'œuvre de la photographe, les images, à la différence de celles du format «coffee table book» retenu par Peter Greenaway, sont légèrement plus grandes que nature, à savoir, dans le cas des deux exemples ci-dessus, $186 \times 70 \mathrm{~cm}$. Bien qu'exceptionnellement l'une des images ait été partiellement traitée numériquement, toutes deux se distinguent des nus de Peter Greenaway par la sobriété et la simplicité de la mise en scène, les jeunes femmes ayant pour seuls attributs de leur identité allégorique la coiffe et l'archet et l'ensemble des signatures.

Que penser de tels choix artistiques ${ }^{12}$ ? Certes, plus puissamment même que les photogrammes de Cindy Sherman, ces femmes nues, les emblèmes de leur sexe servant de blason génétique, allient érotisme et ironie: leur regard fier et défiant engage celui des hommes dont elles savent d'avance qu'ils vont les regarder avec concupiscence. Mais en plus, et c'est particulièrement évident dans le cas de ces exemples, ces corps plus grands que nature ne sont pas simplement photographiés, ils sont comme sculptés dans la photographie par le jeu d'ombre et de lumière grâce au savoir-faire de la photographe: korai des temps modernes, elles revendiquent des valeurs esthétiques et plastiques propres, des valeurs esthétiques et plastiques attribuées dans une culture franchement patriarcale aux kouroi monumentaux des temps passés. En cela, elles figurent non seulement les allégories visées par l'artiste photographe mais, peut-être moins consciemment, l'allégorie de l'esthétique même, de cette appréhension du monde localisée non pas dans la raison mais dans le corps, ici très explicitement dans le corps nu de la femme.

Et la mélancolie et la tête de mort de Benjamin et de Craig Owens dans tout cela? Il est clair que ni le 


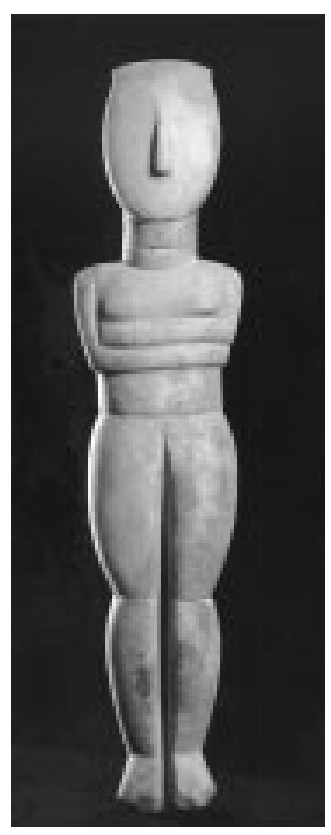

Planche 8. Figurine cycladique de femme, env. 2800-2300 av. J.-C.

regard que porte Peter Greenaway sur son microcosme ni celui que porte Dany Leriche sur le sien ne sont teintés de mélancolie: chez celle-ci, c'est le regard idéologiquement chargé d'une féministe qui renvoie à l'homme l'image d'un monde à l'envers, un monde où c'est la femme qui constitue le repère culturel dominant et où l'homme est invisible. Il est tout aussi clair que ce qui constitue, aussi bien pour Dany Leriche que pour Peter Greenaway, la figure «la plus soumise à l'empire de la nature», autrement dit la caractéristique définitoire de notre humanité, ce n'est pas la tête de mort, mais notre sexualité qui nous oriente, non pas vers le passé mais, au contraire, vers l'avenir de l'espèce, renouant en ceci avec une tradition plus ancienne, peut-être plus optimiste, certainement plus réjouie et plus réjouissante (planche 8).

\section{FORME ET FOND DANS L'IMAGE ALLÉGORIQUE}

S'il est impossible d'identifier une fois pour toutes les traits définitoires et le fond thématique de l'allégorie visuelle, il faut se demander ce qui a garanti la survie du mode depuis sa première définition, il y a plus de vingt siècles ${ }^{13}$. Ce ne peut être que la forme spécifique que le mode présente. De plus, au cours de cette longue évolution, l'allégorie a constamment été associée à la métaphore, voire a longtemps été définie comme une sorte de métaphore filée ${ }^{14}$. Dans cette ultime partie, je voudrais brièvement rendre compte de ce qui me semble être la forme spécifique du mode et des différences qu'il présente par rapport à la métaphore et, par la même occasion, reprendre le problème du supplément.

Reprenons à nouveau l'affiche de Barbara Krüger, dont on a vu qu'elle était informée par la métaphore aussi bien sur le plan textuel que visuel. En paraphrasant à nouveau un peu rapidement la définition de la métaphore que nous lègue C.S. Peirce (dont la sémiotique cherchait à rendre compte non pas des opérations du langage mais de celles du raisonnement), on dira qu'un signe informé par la métaphore a pour fonction de représenter le parallélisme qui structure son objet. Si le signe a été correctement interprété, l'interprétant présentera également la forme d'un parallélisme. Le texte de l'affiche en offre un exemple très simple qu'on représentera par la figure 1 :

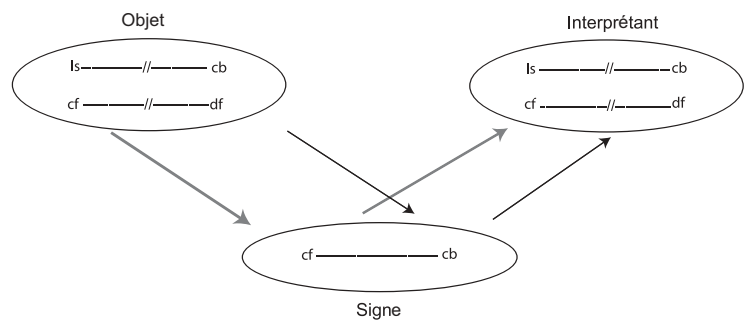

Figure 1. Structure iconique de la métaphore.

La figure montre, en les simplifiant, les relations de détermination qui "partent" de l'objet et qui se terminent dans l'interprétant, en passant par le signe: la détermination de l'interprétant par l'objet est ainsi médiatisée par le signe. Dans l'objet, le parallélisme du texte de Krüger met en relation les deux éléments suivants: le lieu stratégique (ls) et le champ de bataille (cb), qui constituent ainsi les éléments du domaine de base, de ce donné qui sert de point de départ au jugement «métaphorique». En revanche, le corps de la femme, l'enjeu de cette bataille, est représenté dans l'objet par l'élément cf, alors que les droits de la femme, y compris le droit d'avorter si elle le veut, sont représentés par df. Ces deux éléments constituent ce que l'on convient d'appeler la cible de la métaphore, c'est-à-dire le domaine d'expérience problématique sur 
lequel porte le jugement. La figure illustre en même temps une deuxième caractéristique de la structure hypoiconique de tout signe, scriptural ou pictural: alors que l'objet est immatériel, pensé, le signe pour être perçu doit se manifester physiquement en passant par un médium existentiel, ici quelques lettres inscrites sur une affiche. Le passage de la pensée à la manifestation physique écrite entraîne une perte d'informations: aucun signe, qu'il soit linéaire ou bidimensionnel, ne peut représenter hiérarchiquement tous les éléments du parallélisme, car on passe de la catégorie complexe de la tiercéité (la pensée) à la catégorie nettement moins complexe de l'existence, c'est-à-dire à la secondéité. C'est pourquoi le signe, lui, n'asserte que la mise en relation des éléments cf et cb: Your body is a battleground. Enfin, si nous avons correctement inféré la signification du texte, le parallélisme initial s'inscrira dans l'interprétant. Autrement dit, notre comportement, en voyant l'affiche, intégrera forcément la structure métaphorique de l'objet que le signe ne peut représenter que partiellement.

Qu'en est-il de l'allégorie? Il a été suggéré plus haut que, d'une certaine façon, l'affiche de Barbara Krüger pouvait s'interpréter comme l'allégorie de la manière dont le linguistique, au cours du siècle dernier, a pris le dessus sur la perception visuelle dans notre manière occidentale d'appréhender le monde. S'agissant d'allégorie, la tâche d'interprétation est plus complexe que dans le cas d'une image métaphorique. La figure 2 montre clairement pourquoi il en est ainsi:

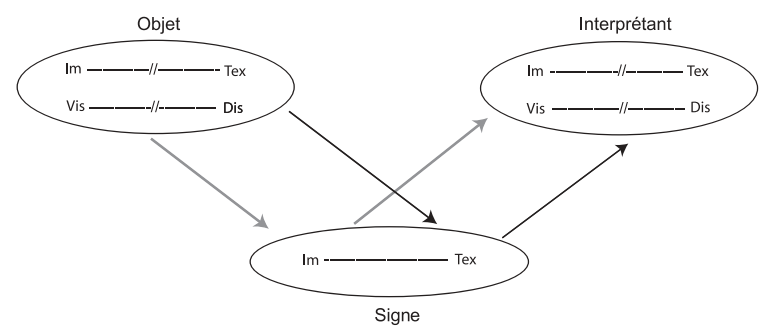

Figure 2. Structure métaphorique de l'allégorie.

Alors que le signe linguistique Your body is a battleground incorpore des éléments de la base et de la cible, à savoir battleground et body respectivement, dans le cas de l'allégorie, le signe constitué par l'affiche entière ne comporte que les éléments de la base: ici, l'élément pictural (Im) qui, sur l'affiche, se trouve subordonné au texte (Tex), laissant ainsi à l'interprète la tâche d'inférer les éléments de la cible, qui sont respectivement la perception visuelle (Vis) - cet ancien fondement de notre métaphysique occidentale qui est en train de céder du terrain devant la montée du tournant linguistique -, lequel est figuré dans le parallélisme par le discours (Dis). Tâche difficile, certes, mais nullement impossible, puisqu'elle est déjà inscrite justement comme une possibilité dans la structure hypoiconique de la métaphore. Telle qu'elle se définit ici donc, l'allégorie se caractérise par la manière dont l'observateur est contraint, en s'en remettant à des informations parfois fort elliptiques et allusives (du fait que la structure métaphorique qui informe toute allégorie perd forcément des informations, une fois exposée aux contraintes d'un médium existentiel), d'inférer, à partir des éléments fournis par la base, l'identité de la cible «inscrite» dans le parallélisme qui structure l'objet du signe. L'allégorie se distingue par conséquent de la métaphore filée moins par sa forme - il s'agit de la métaphore au sens formel peircien dans les deux cas ${ }^{15}$ - que par sa portée, car la structure de l'allégorie et sa façon de ne représenter que les éléments de la base sont coextensives avec l'ensemble de l'affiche, et non pas avec une quelconque de ses parties.

Nous sommes maintenant en mesure de revenir sur le problème du statut «supplémental», qu'Owens a dévolu à l'allégorie, derrière lequel se profile l'un des concepts clés de la grammatologie. Comme Owens confond volontairement allégorie et allégorèse dans son étude, c'est-à-dire l'idée qu'un premier texte est interprété par un texte écrit ultérieurement, et comme il tient pour acquise l'idée qu'un phénomène forcément linéaire et unidimensionnel comme un texte puisse servir de modèle au signe bidimensionnel qu'est l'image, on comprend mieux ce glissement qui conçoit comme rapport de supplémentarité ce que l'hypoiconicité métaphorique représente 
«verticalement» comme un parallélisme. Tout ce qui se rapporte à un texte originaire en est forcément une sorte de commentaire, un supplément d'information, donc ${ }^{16}$. Ce qui n'en fait pas forcément une allégorie. En revanche, si l'on adopte une position franchement visualiste comme celle qui a engendré la théorie peircienne de l'hypoiconicité, à savoir une conception de la structure qui, l'affiche de Barbara Krüger le montre clairement, vaut aussi bien pour un texte que pour une image, il est possible d'éviter les pièges réducteurs du «tout texte», du «tout écrit» ou du "tout discours", du genre Langages de l'art, et de respecter, de la sorte, la spécificité des divers genres littéraires ou artistiques soumis à l'analyse.

\section{CONCLUSION}

L'identification de l'allégorie est un problème subjectif, ou pour être plus précis, une question d'idéologie. Notre capacité à débusquer l'allégorie a plus de chances d'être déterminée par des attentes culturellement motivées que par une quelconque perspective personnelle. (Laird, 2003: 153; trad. T.J.)

Le problème est là: l'allégorie, son étymologie le montre clairement, a débuté dans la pensée occidentale comme une façon de parler et, sous le nom d'vлovoı $\alpha$, comme une stratégie interprétative ${ }^{17}$. Plus tard, elle a fait son entrée dans le monde des images, lesquelles par la suite, sans doute à cause du caractère forcément rhématique de toute représentation exclusivement picturale, se sont affublées d'un texte explicatif. Sa fonction a de ce fait évolué et s'est diversifiée, son fond emblématique aussi. Le signe allégorique pictural étant rhématique, ne sont visibles que des éléments parfois hétérogènes, mais rarement aléatoires, que l'interprète cherche à «sommer» dans une interprétation cohérente qui dépend entièrement de son expérience culturellement déterminée, comme l'a bien compris Laird dans le texte cité plus haut. Ce qui rend problématique toute circonscription du mode et toute tentative d'en établir une synthèse une fois pour toutes. Owens a procédé par un examen du fond de l'allégorie, se justifiant dans cette tentative par un recours à une vision linguistique des phénomènes sémiotiques. Les quatre exemples d'allégories photographiques contemporaines examinés plus haut ne lui ont pas donné raison.

Par conséquent, il me semble que c'est par la forme, par l'uniformité même, que présente l'allégorie, mieux que par "ce modèle énigmatique de la ligne», qu'on peut davantage saisir la spécificité du mode, car la thématique et les objectifs mêmes déterminant le recours au mode allégorique peuvent, n'en déplaise à Owens et à Benjamin, varier considérablement d'une période à une autre, et varier même géographiquement à une période donnée ${ }^{18}$.

Nous venons de passer rapidement en revue quatre images allégoriques qui exploitent respectivement des thèmes mythologiques, païens, images qui ont été créées à des fins esthétiques et militantes. On comprend bien que. dans ces cas, ce n'est ni le but poursuivi ni leur contenu thématique respectif qui est invariant, mais la forme métaphorique à partir de laquelle les images ont été construites.

\section{N O TES}

1. Le texte de C. Owens, «The Allegorical Impulse: Toward a Theory of the Postmodernism ", a été publié dans Wallis et Tucker $(1984: 203$ 253) (éd. originale en 1980 dans October, no 12 , p. $67-86$ et no 13 , p. 59 80). Précisons que c'est moi qui traduis les passages cités. Il est à noter que le texte d'Owens appartient à une série d'études théoriques visant à redorer la réputation quelque peu ternie de l'allégorie au XXe siècle. Voir, par exemple, Whitman (1987).

2. Pour emprunter une terminologie employée par la critique anglosaxonne.

3. "Spectacle/drame du deuil», donc "tragédie».

4. Il est à noter que Benjamin précise bien qu'il s'agit d'un culte

baroque: "Les allégories sont au domaine de la pensée ce que les ruines sont au domaine des choses. D'où le culte baroque de la ruine"

(Benjamin, 1985: 191).

5. C'est dans le cadre d'un "Grand Atelier» qui a eu lieu en 1995 à l'invitation de l'Université des sciences humaines de Strasbourg que Greenaway a entrepris de ramener à cent un ensemble d'allégories susceptibles de «dresser, en cette fin de millénaire, à partir de modèles anciens ou récents, un nouvel inventaire de cent allégories pour représenter le monde». Plus de cent cinquante habitants de Strasbourg - modèles bénévoles, universitaires, étudiants, etc. - ont accepté de poser nus devant deux photographes. Les images ainsi obtenues ont par la suite été traitées numériquement par la société Andromaque, sous la direction artistique de Peter Greenaway, qui les a «habillées» en y ajoutant des éléments de son propre travail d'artiste peintre, ainsi que des emprunts à une liste de "précurseurs » qui comprend, entre autres, 
le photographe Eadweard Muybridge, l'encyclopédiste Diderot, l'astrologue et mystique Robert Fludd et l'égyptologue jésuite Athanasius Kircher; aux efforts desquels, «en vue d'inventorier, de collationner et de regrouper les savoirs en vue de mettre le monde en livre », Greenaway déclare avoir voulu rendre hommage (Greenaway, 1998: 7). Cette collaboration entre professionnels de l'image et modèles volontaires s'est concrétisée par la publication de cet ouvrage, où les cent allégories sont regroupées en vingt-deux «familles " d'allégories plus ou moins traditionnelles (figures mythiques comme le Jour et la Nuit, des Muses comme la Danse et la Peinture, ainsi que des allégories plus personnelles comme les Moteurs de l'univers, Europa et Junon, par exemple) numérotées de un à cent. À la différence des livres d'emblèmes d'Alciat ou de Ripa, par exemple, les textes (bilingues), qui normalement complémentent les images, ont ici été relégués en fin d'ouvrage, donnant forcément une idée de la supériorité accordée par l'auteur au visuel par rapport au discursif.

Les images de Greenaway (Marsyas et La Destinée) sont publiées ici sous réserve de l'approbation des ayants droit. Nous avons tenté auprès de plusieurs organismes d'obtenir les droits d'utilisation, mais en vain. Nous invitons les titulaires des droits à nous contacter afin de régulariser la situation.

6. Les prises de vue remontent à 1995.

7. «On peut en gros diviser les hypoicones suivant le mode de priméité auquel elles participent. Celles qui font partie des simples qualités ou premières priméités sont des images; celles qui représentent les relations, principalement dyadiques ou considérées comme telles, des parties d'une chose par des relations analogues dans leurs propres parties, sont des diagrammes; celles qui représentent le caractère représentatif d'un representamen en représentant un parallélisme dans quelque chose d'autre sont des métaphores.» (Peirce, 1978: 2.277).

8. Il cite un passage de l'Esthétique de Benedetto Croce.

9. Je tiens à remercier Dany Leriche de m'avoir autorisé à me servir des allégories Anoki et Sophie.

10. Le commentaire de Jeanne Eloi, esthéticienne qui a participé à la sélection des thèmes à illustrer, fourni post factum à l'allégorie Virilité de Dany Leriche (reproduite dans l'article de Mirelle Thijsen du présent dossier), permet de mesurer toute la teneur idéologique du projet: "Virilité est une femme, nue et voluptueuse. Elle est étendue sur une couche formée d'un amoncellement de cadres dorés, dont les lignes emplissent les trois quarts d'une chambre étroite. À ses pieds, se trouve un coq dont la crête touche le bord de la pièce. Les cadres superposés représentent l'histoire des représentations. Ils forment un paysage qui ennuie l'œil par sa répétition et maintient la femme couchée. C'est ici que l'homme la traque sous la forme d'un coq à la crête épanouie. Mais bientôt viendra le siècle où, privé d'espace, le volatile repliera ses atours et adoptera de nouvelles postures. Convaincu que femme et nature ne faisaient qu'un, l'homme s'est comporté en animal; laissant apparaître l'impulsion qu'un être vivant doit à ses organes, il a assigné à la femme la pose qui justifiait sa possession. Renversant les rôles, il a soumis l'histoire à sa loi. C'est pourquoi Virilité est elle-même assujettie à une représentation historique - image figée déjà lointaine, mais présente encore dans nos mémoires" (Les Filles de Ripa, p. 42). 11. Et ce n'est pas par simple conformité à une tradition déterminée essentiellement par le genre grammatical du concept, la fraude, par exemple, qui fait que les personnifications féminines prédominent, et que Dany Leriche choisit de figurer ces allégories par des femmes (voir Warner, 1985: chap. 4; Leidl, 2003: 31-54).

12. Pour une discussion d'une allégorie de Leriche ne faisant pas partie de la série des Filles de Ripa, voir Jappy (2005).

13. On ne trouve aucune instance du terme "allégorie» avant le troisième siècle avant notre ère (ou le premier selon la datation que l'on accepte en ce qui concerne le rhétoricien Demetrius). 14. Voir l'annexe I de Whitman (1987) pour une excellente discussion de la conception antique des rapports complexes entretenus par l'allégorie et la métaphore; ou encore Innes (2003: 18-19). Le problème a été résolu plus simplement par Bierce (1967).

15. Autrement dit, la métaphore est une structure hypoiconique dont l'allégorie, qu'elle soit scripturale ou picturale, est l'une des réalisations.

16. "Conceived [as a palimpsest] allegory becomes the model of all commentary, all critique, insofar as these are involved in rewriting a primary text in terms of its figural meaning"(Owens, 1984: 205).

17. Voir le recueil d'articles de Boys-Stones (2003).

18. Voir T. Jappy (2005) pour une discussion plus développée.

\section{RÉFÉREN CES BIBLIOG RAPHIQ U ES}

BENJAMIN, W. [1985]: Origine du drame baroque allemand, Paris, Flammarion (éd. originale: The Origin of the German Tragic Drama, Londres, New Left Books, 1977).

BIERCE, A. [1967]: The Devil's Dictionary, Harmondsworth, Penguin ("Allegory: A metaphor in three volumes and a tiger»).

BOYs-STONES, G. R. (dir.) [2003]: Metaphor, Allegory and the Classical

Tradition: Ancient Thought and Modern Revisions, Oxford, OUP. CLARK, K. [1969]: Civilisation, Londres, John Murray.

CusseT, F. [2003]: French Theory. Derrida, Foucault, Deleuze et Cie: l'aventure américaine des intellectuels français, Paris, La Découverte, 38-42. DERRIDA, J. [1967]: De la grammatologie, Paris, Minuit.

EDWARDS, M. [2003]: "Origen on Christ, Tropology, and Exegesis", dans G. R. Boys-Stones, 235-256.

GASQUET, L. [2003]: «Peter Greenaway's Allégories or the Ethics of Systems ", Études britanniques contemporaines: revue de la société d'études anglaises, Montpellier, $\mathrm{n}^{\circ} 65$.

GREENAWAY, P. [1998] : Cent allégories pour représenter le monde, Paris, Éd. Adam Biro.

INNES, D. [2003] : «Metaphor, Simile, and Allegory as Ornament of Style ", dans G. R. Boys-Stones, 18-19.

JAPPY, T. [2005] : «Allegory, Metaphor and Medium », à paraître dans Visio, vol. 9, nos 3-4, automne-hiver.

JAY, M. [1994]: Downcast Eyes: The Denigration of Vision in Twentieth Century French Thought, Berkeley, University of California Press. LAIRD, A. [2003]: «Figures of Allegory from Homer to Latin Epic», dans G. R. Boys-Stones, 151-175.

LEIDL, C. [2003]: «Metaphor and Literary Criticism », dans G. R. BoysStones, 31-54.

OWENS, C. [1984]: «The Allegorical Impulse: Toward a Theory of the Postmodernism", dans B. Wallis et M. Tucker, 203-235.

PeirCe, C. S. [1931-1958]: Collected Papers, 4 vols., sous la dir. de C. Hartshorne, P. Weiss et A. Burks, Cambridge (Mass.), Harvard University Press:

[1978] : Écrits sur le signe, (rassemblés, traduits et commentés par G. Deledalle), Paris, Le Seuil.

PorCheron, M.-D. [2000]: Dany Leriche. Les Filles de Ripa, préface au catalogue, Paris, Galerie Rachlin-Lemarié.

WALLIS, B. et M. TUCKER (dir.) [1984]: Art after Modernism: Rethinking representation, New York, New Museum of Contemporary Art.

WARNER, M. [1985]: Monuments and Maidens: The Allegory of the Female Form, Londres, Picador.

Whitman, J. [1987]: Allegory: The Dynamics of a Ancient and Medieval Technique, Cambridge (Mass.), Harvard University Press. 